\title{
Development of an immunotherapeutic adenovirus targeting hormone-independent prostate cancer
}

\author{
Jae Sik Kim' \\ Sang Don Lee ${ }^{2}$ \\ Sang Jin Lee ${ }^{3}$ \\ Moon Kee Chung ${ }^{2}$ \\ 'Department of Urology, The Catholic \\ University of Korea Incheon St Mary's \\ Hospital, Incheon, ${ }^{2}$ Pusan National \\ University Yangsan Hospital and \\ Research Institute for Convergence \\ of Biomedical Science and Technology, \\ Yangsan, ${ }^{3}$ Genitourinary Cancer \\ Branch, National Cancer Center, \\ Goyang, Korea
}

This article was published in the following Dove Press journal:

OncoTargets and Therapy

II November 2013

Number of times this article has been viewed
Background: To develop a targeting therapy for hormone-independent prostate cancer, we constructed and characterized conditionally replicating oncolytic adenovirus (Ad) equipped with mRFP (monomeric red fluorescence protein)/ttk (modified herpes simplex virus thymidine kinase). This construct was then further modified to express both $\mathrm{mRFP} / \mathrm{ttk}$ and a soluble form of cytokine FLT3L (fms-related tyrosine kinase 3 ligand) simultaneously.

Methods: To construct the recombinant oncolytic adenovirus, E1a and E4 genes, which are necessary for adenovirus replication, were controlled by the prostate-specific enhancer sequence (PSES) targeting prostate cancer cells expressing prostate-specific antigen (PSA) and prostatespecific membrane antigen (PSMA). Simultaneously, it expressed the $\mathrm{mRFP} / \mathrm{ttk}$ fusion protein in order to be able to elicit the cytotoxic effect.

Results: The Ad5/35PSES.mRFP/ttk chimeric recombinant adenovirus was generated successfully. When replication of Ad5/35PSES.mRFP/ttk was evaluated in prostate cancer cell lines under fluorescence microscopy, red fluorescence intensity increased more in LNCaP cells, suggesting that the $\mathrm{mRFP} / \mathrm{ttk}$ fusion protein was folded functionally. In addition, the replication assay including wild-type adenovirus as a positive control showed that PSES-positive cells (LNCaP and CWR22rv) permitted virus replication but not PSES-negative cells (DU145 and PC3). Next, we evaluated the killing activity of this recombinant adenovirus. The Ad5/35PSES. $\mathrm{mRFP} / \mathrm{ttk}$ killed LNCaP and CWR22rv more effectively. Unlike PSES-positive cells, DU145 and PC3 were resistant to killing by this recombinant adenovirus. Finally, in order to potentiate therapeutic efficacy, we developed a recombinant adenovirus expressing multiexogenous genes, $\mathrm{mRFP} / \mathrm{ttk}$ and sFLT3L.

Conclusion: In the present study, a replication-competent adenovirus was successfully designed to replicate conditionally in PSA-positive and PSMA-positive prostate cancer cells. This recombinant adenovirus is equipped with the fusion protein of suicidal and red-fluorescence fusion protein together with sFLT3L. This construct would be expected to have potent antitumor effects and deserves more extensive investigation.

Keywords: adenovirus, prostate cancer, hormone-independent, suicide gene

\section{Introduction}

In 2008, prostate cancer was the fifth most commonly diagnosed malignancy in males in Korea, with approximately 6,471 men (7\%) presenting as new cases. ${ }^{1}$ The rate of diagnosis of prostate cancer was $13.5 \%$. This prostate cancer detection rate is increasing in Korea. The death rate due to prostate cancer was $0.5 \%$ in 1990 , but was $2.4 \%$ in 2008. Although most patients are diagnosed as having local organ-confined disease at the first doctor's visit, some patients present with locally advanced disease or detectable bone metastasis. The only treatment modality available for patients with advanced
Correspondence: Moon Kee Chung Department of Urology, Pusan National University Yangsan Hospital, Beomeo-ri, Mulgeum-eup, Yangsan-si 626-770, Korea Tel +82 553602134

Fax +82 553602164

Email mkchung@pusan.ac.kr 
metastatic prostate cancer is androgen ablation therapy. In general, hormone therapy induces remission in 80\%-90\% of men, and holds cancer growth in check for an average of 2-3 years. However, tumor regression is transient, and the disease inevitably progresses to androgen-independent status. Therefore, no effective therapy is available to treat prostate cancer, so the disease becomes lethal. This study applied gene therapy, based on a decade of accumulated knowledge and recent breakthrough information, to generate a novel therapeutic agent and establish a treatment modality for the disease.

\section{Materials and methods Construction and generation of recombinant adenovirus}

In brief, we used two plasmids for construction of the adenovirus: the cloning shuttle vector harboring the adenoviral left intron region and packaging signal and the rest of the adenoviral genome vector containing the right arm of the adenoviral genome; and the cloning shuttle vector containing E1aTATA described by Ali. ${ }^{2}$ Next, we inserted E4TATA. Finally, in order to enable the E1a under the prostate-specific promoter, the prostate-specific enhancer sequence (PSES) ${ }^{3}$ was inserted into a vector. A mRFP/ttk fusion protein expression cassette was cloned. PSES-mRFP/ttk release together with the adenoviral left intron region. The plate was incubated at $37^{\circ} \mathrm{C}$ under $5 \% \mathrm{CO}_{2}$, until plaques became large enough to be isolated. The amplified adenoviruses were purified by $\mathrm{CsCl}$ gradient centrifugation. ${ }^{4}$

\section{Flow cytometry analysis and thymidine kinase enzymatic assay}

LNCaP, CWR22rv, PC-3, and DU145 cells were seeded at a density of $1 \times 10^{6}$ cells in six-well plates and subsequently infected with recombinant adenovirus. The medium was changed 24 hours later, and the cells were examined by flow cytometry 24 hours post viral infection or under the microscope daily for up to 5 days to see red fluorescence protein for thymidine kinase fusion protein ( $\mathrm{mRFP} / \mathrm{TK}$ ) expression. Herpes simplex virus thymidine kinase enzyme activity of wild-type adenovirus or Ad5/35PSES.mRFP/ttk was determined by measuring the amount of phosphorylated GCV accumulated in the cells. Briefly, cells at a density of $3 \times 10^{5}$ were infected with one multiplicity of infection at $37^{\circ} \mathrm{C}$ for 24 hours and then incubated in the presence or absence of $\left[8^{-3} \mathrm{H}\right] \mathrm{PCV}(1.0 \mu \mathrm{Ci} / \mathrm{mL})$. After 2 hours, the radioactivity in the medium and cells was measured. The cultured wells were washed with phosphate-buffered saline and pelleted briefly for 2 minutes to harvest the cells. The radioactivity in the cell pellets was determined. Triplicate samples were used in all uptake assays. Protein concentrations in the cell lysates from the same wells were also determined for normalization.

\section{Viral replication assay}

LNCaP, CWR22rv, PC-3, and DU145 cells were seeded in six-well plates and subsequently infected with recombinant adenovirus. Each cell line was infected with 100-400 virus particles per cell depending on infectivity. The medium was changed one day later, and the viral supernatants were harvested 3 days after infection. The cells were examined under the microscope daily for up to 5 days.

\section{In vitro cytotoxicity assay}

LNCaP, CWR22rv, PC-3, and DU145 cells were seeded in 96-well plates and infected with serial doses, ranging from 5 to $5 \times 10^{-9}$ multiplicity of infection of recombinant adenovirus. A row of six wells seeded with each type of cell was used for each dose. The medium was refreshed on day 4 , and the cells were examined under a light microscope on day 7 to determine the adenovirus-mediated cytopathic effects. A killing activity index was obtained. ${ }^{5}$

\section{Western blotting analysis}

LNCaP cells at a density of $4 \times 10^{5}$ were seeded in six-well plates and infected with recombinant adenovirus Ad5/35sFLT3L. PSES.mRFP/ttk for 48 hours. The cells were then lysed with radioimmunoprecipitation assay buffer containing protease inhibitors (Sigma-Aldrich, St Louis, MO, USA). Proteins of $80 \mu \mathrm{g}$ were resolved by sodium dodecyl sulfate polyacrylamide gel electrophoresis and transferred to a polyvinylidene fluoride membrane (Amersham Life Science, Arlington Heights, IL, USA). Membranes were probed with antibodies against FLT3L (Santa Cruz Biotechnology Inc., Santa Cruz, CA, USA), and the subsequent corresponding secondary antibody (Jackson Immuno Research Laboratories, West Grove, MA, USA) was visualized using the Enhanced Chemiluminescence (ECL) Plus kit (Amersham Life Science).

\section{Results}

\section{Construction of adenovirus expressing $\mathrm{mRFP} / \mathrm{ttk}$ controlled PSES promoter}

In this study, the recombinant adenovirus was constructed to replicate in PSES-positive cells, which express both PSA and PSMA (Figure 1). To control adenovirus replication in PSESpositive cells, PSES was inserted to control adenoviral E1a and $\mathrm{E} 4$ protein expression. ${ }^{5}$ At the right end of the adenoviral 


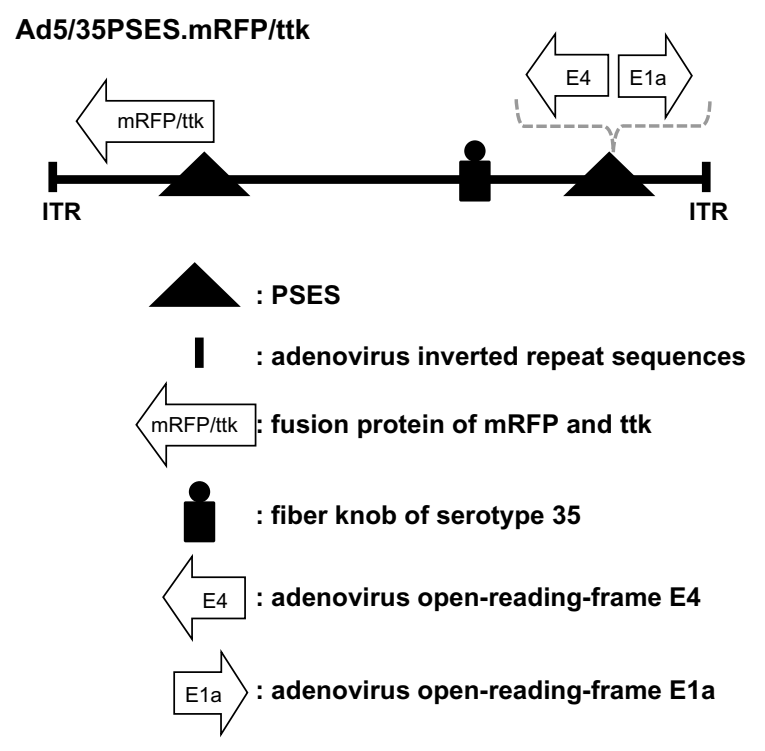

Figure I Structure of the recombinant adenovirus (Ad5/35PSES.mRFP/ttk). The PSES promoter allows replication of the virus in prostate cancer cells by controlling early Ela and E4 viral gene expression. PSES also drove mRFP/ttk gene expression at the right end.

Abbreviations: Ad, adenovirus; PSES, prostate specific enhancer sequence; ITR, intron, mRFP, monomeric red fluorescence protein; ttk, modified herpes simplex virus thymidine kinase.

genome, the $\mathrm{mRFP} / \mathrm{ttk}$ cytotoxic gene was inserted under the control of another copy of PSES. mRFP/ttk was the fusion protein composed of monomeric red fluorescence protein (mRFP) and the modified herpes simplex virus thymidine kinase, which is known to have a higher enzymatic activity than thymidine kinase.

Although most of the adenoviral vectors used in gene therapy have adopted serotype 5, we made a chimeric adenovirus Ad5/35, which had the serotype 35 fiber knob on the serotype 5 backbone. Serotype 35 binds to cellular CD46 as a receptor as opposed to the Coxsackievirus-adenovirus (CAR) receptor for serotype $5 .{ }^{6}$ This chimeric recombinant adenovirus could presumably overcome the limitation by the low CAR expression level shown in several solid tumors. ${ }^{6,7}$

\section{Thymidine kinase enzymatic assay and flow cytometry analysis for mRFP}

We detected the expression of red fluorescence protein by flow cytometer in LNCaP and CWR22rv, which are PSA-positive and PSMA-positive cells. Compared with no virus particles as a negative control, adenovirus Ad5/35PSES.mRFP/ttk mediated high expression of mRFP (Figure 2A). Consistent with Figure 2A, as shown in Figure 2B, greater accumulation occurred in LNCaP and CWR22rv cells but not in PC3 and DU145 cells as expected. These results were consistent with the fact that PSES was demonstrated to be active in LNCaP and CWR22rv cells but not in PC3 and DU145 cells. ${ }^{3}$

\section{Virus replication in PSES-positive cells}

To test whether PSES could control viral replication, we infected LNCaP cells with different numbers of recombinant adenovirus and monitored $\mathrm{mRFP}$ gene expression at different time points for up to 5 days (Figure 3 ). The red fluorescence intensity increased with increasing incubation time and more virus particles produced bright red fluorescence, suggesting fusion protein expression. At the same time, as shown in pictures taken under light microscopy, there was an extensive cytopathic effect due to virus replication.

To address whether this recombinant adenovirus could replicate in prostate cancer cells, we investigated its replication efficiency in PSES-negative cells (DU145 and PC3) and PSES-positive cells (LNCaP and CWR22rv). As shown in Figure 4, the recombinant adenovirus replicated efficiently only in PSES-positive cells.

\section{In vitro cytotoxicity assay}

Figure 5 shows the virus doses which had a cytopathic effect. The recombinant adenovirus was able to kill PSES-positive prostate cancer cells more effectively, consistent with the PSES promoter activity previously reported. ${ }^{3}$ The killing potency of this adenovirus in $\mathrm{LNCaP}$ cells was 10,000 -fold or 1,000-fold higher than in DU145 or PC3 cells.

\section{Construction of immunotherapeutic adenovirus}

As seen in Figure 6, we constructed adenovirus containing both sFLT3L ligand and $\mathrm{mRFP} / \mathrm{ttk}$ as the therapeutic genes. sFLT3L is known to mobilize and stimulate natural killer cells as well as dendritic cells, resulting in an antitumoral action and prolonged patient survival. CWR22 $\mathrm{rv}$ and $\mathrm{LNCaP}$ cells secreted a quite amount of sFLT3L after adenovirus infection.

\section{Discussion}

Androgen ablation is currently the most effective therapy available for patients with advanced prostate cancer. However, androgen ablation therapy does not stop progression of prostate cancer to the lethal androgen-independent state. Unfortunately, there is no effective therapeutic modality available at this moment when androgen-independent prostate cancer emerges. In this study, we applied gene therapy, based on a decade of accumulated knowledge and recent breakthrough information, to generate a novel therapeutic agent and to establish a treatment modality for this disease. Our successful results should be able to be applied to other cancers using other tissue-specific promoters, generating a new 
A

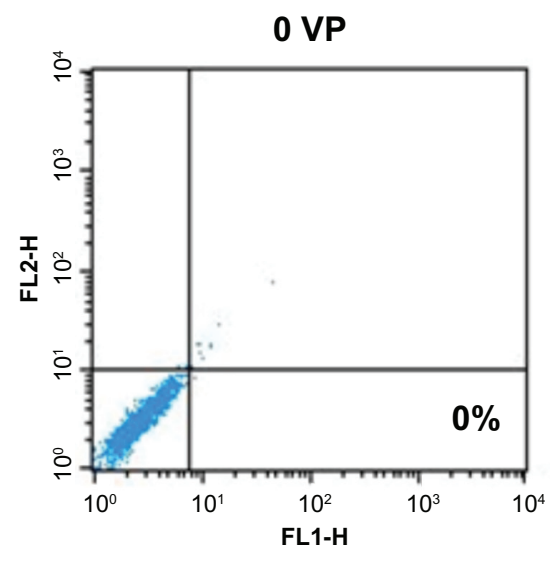

O VP

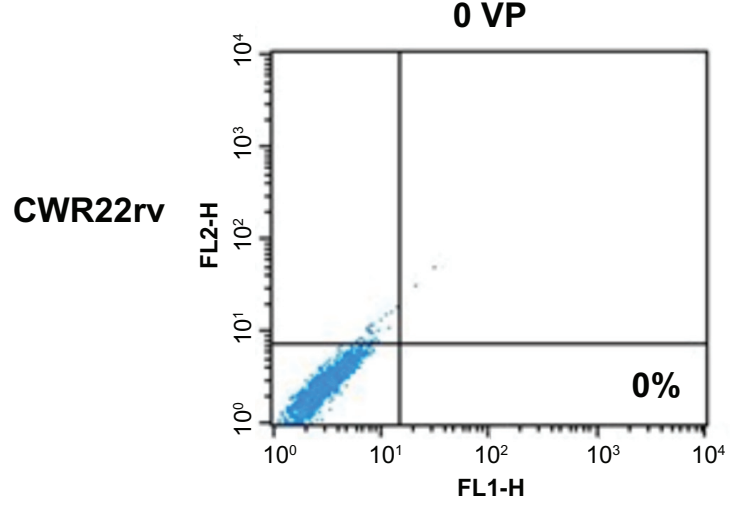

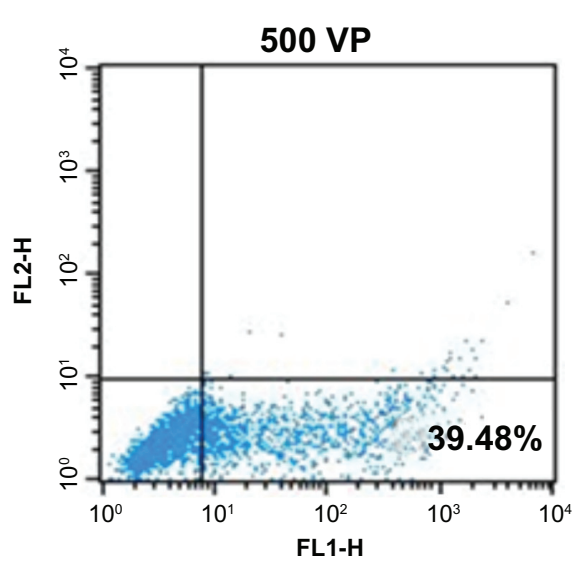

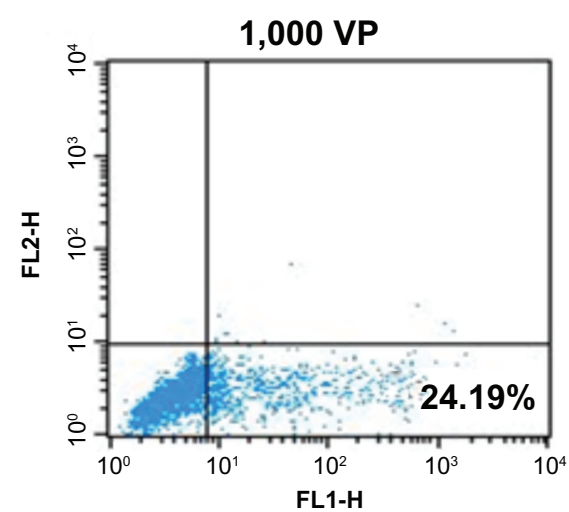

B

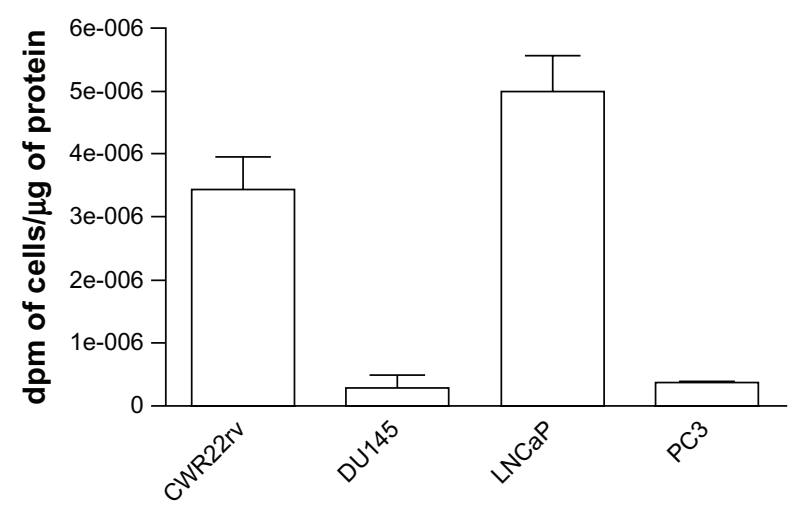

Figure 2 Evaluation of mRFP/ttk expression mediated by Ad5/35PSES mRFP/ttk in prostate cancer cells. (A) Cells were infected with 500 virus particles (LNCaP cells) or I,000 virus particles (CWR22rv cells). After 24 hours, cells expressing red fluorescence were detected by flow cytometry. (B) Cells at a density of $5 \times 10^{6}$ were infected with Ad5/35 mRFP/ttkElaPSESE4. The enzymatic activity of thymidine kinase driven by adenovirus 24 hours post infection was measured as described in the Materials and methods section.

Abbreviations: Ad, adenovirus; dpm, disintegrations per minute; VP, virus particle; mRFP, monomeric red fluorescence protein; ttk, modified herpes simplex virus thymidine kinase; PSES, prostate-specific enhancer sequence.

approach to the treatment of cancer and eventually opening a new door in the field of cancer gene therapy research.

Gene therapy directed to tumor cells provides a promising opportunity to overcome this formidable challenge. Ideally, a well designed gene therapy technique would become a "silver bullet" selectively targeting cancer cells and altering their internal functions to block further growth or triggering lysis. Prostate cancer has been targeted by gene therapeutics since their inception and prostate cancer accounts for $10 \%$ of the gene therapy protocols approved by the Office of Biotechnology Activities in the US. ${ }^{8}$ These approaches have included immune-based, toxic, and oncolytic gene therapies that take advantage of several characteristics specific to prostate cancer. Prostate cancer is ideal for cancer gene therapy for several reasons: the prostate is a nonvital organ that becomes vestigial after the reproductive years; targeting 
A

500 VP

1 day
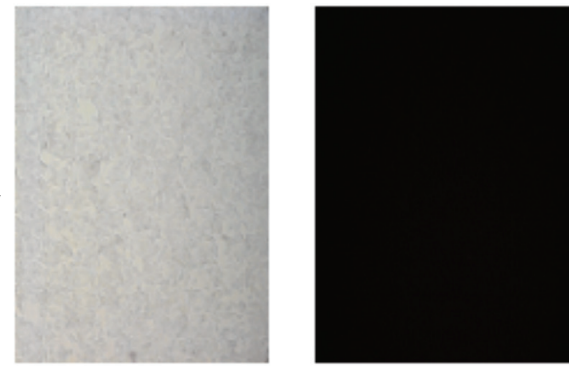

3 days
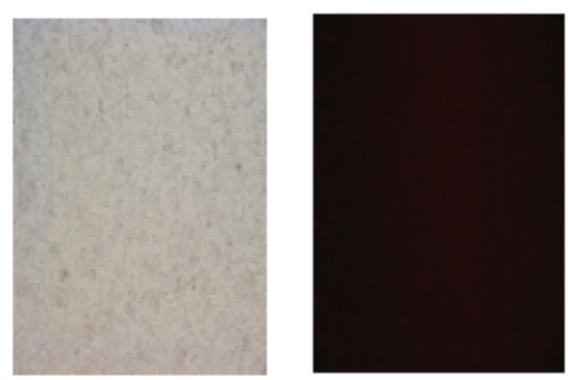

5 days
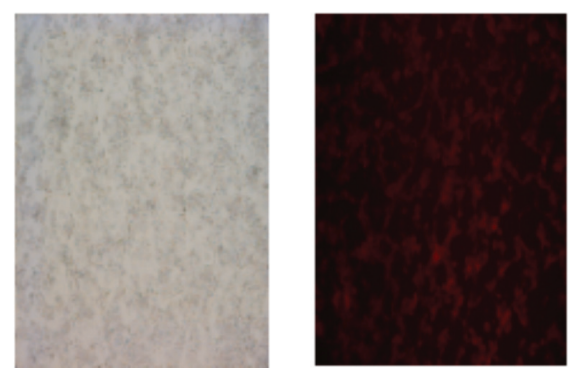

B

$1,000 \mathrm{VP}$

1 day

3 days
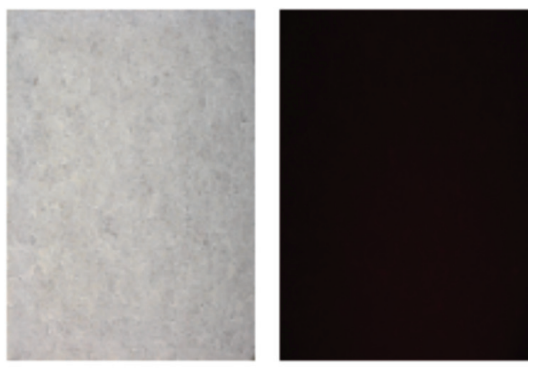

5 days
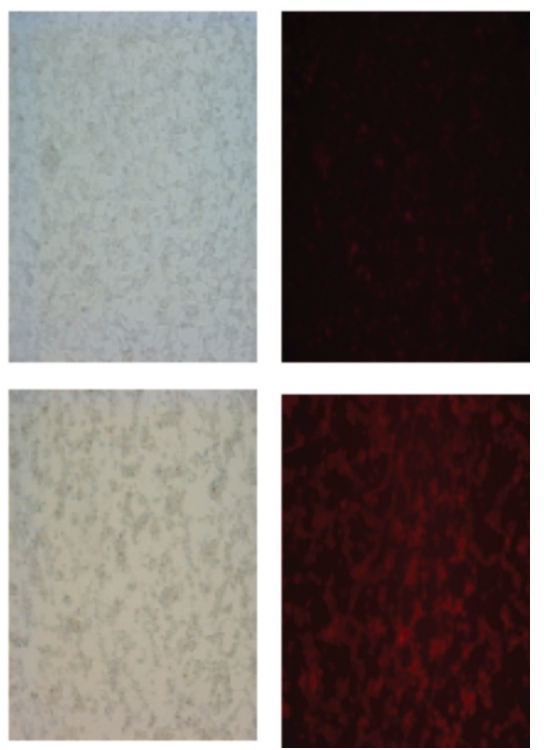

Figure 3 Replication ability of recombinant adenovirus Ad5/35PSES.mRFP/ttk. LNCaP cells were infected with 500 (A) or I,000 (B) virus particles of recombinant adenovirus and monitored for 5 days after infection under fluorescent microscopy and light microscopy (20x).

Abbreviations: Ad, adenovirus; mRFP, monomeric red fluorescence protein; ttk, modified herpes simplex virus thymidine kinase; PSES, prostate-specific enhancer sequence.

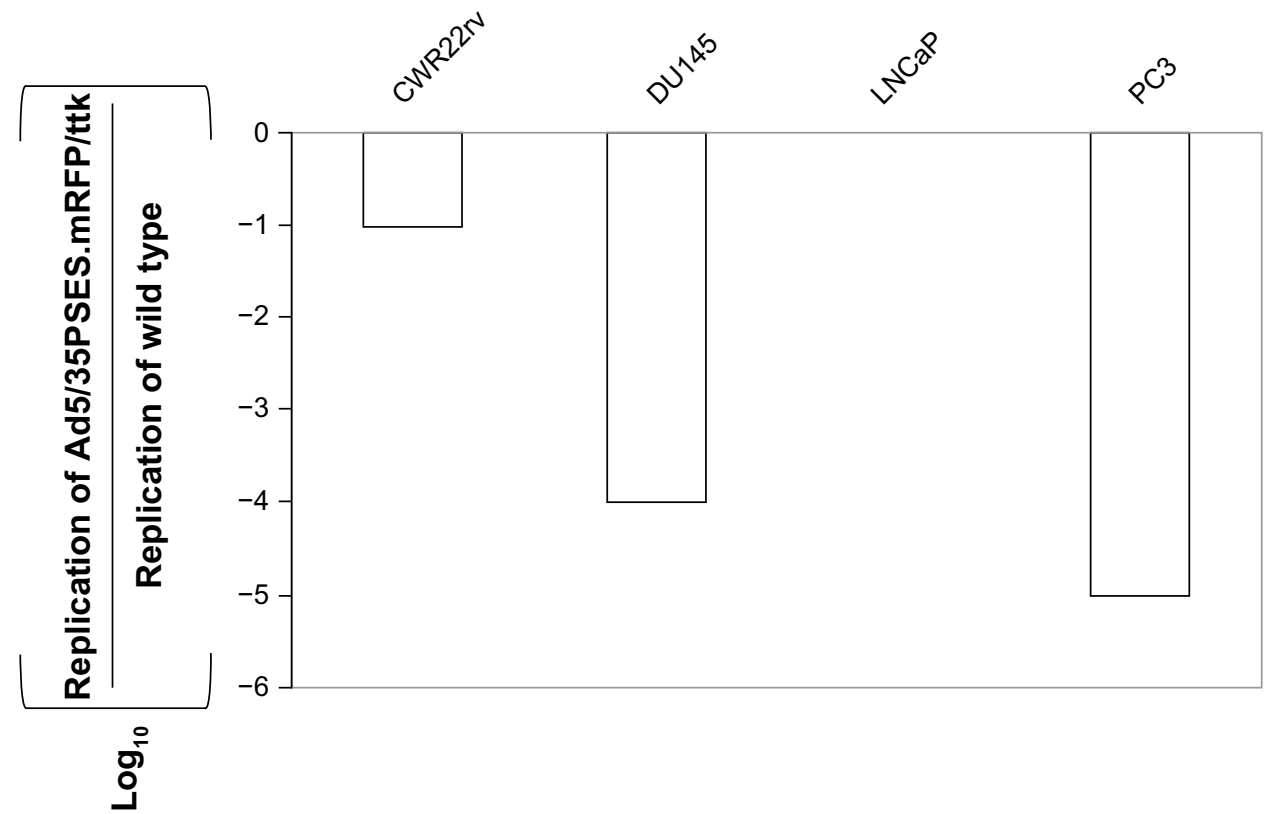

Figure 4 Replication of recombinant adenovirus in several human prostate cancer cell lines in vitro. Cells at a density of $\mathrm{I} \times 10^{6}$ were infected with 200 virus particles of wild-type or Ad5/35PSES.mRFP/ttk per cell. Viruses recovered from the cells and medium 5 days later were titered on confluent layers of 9 I IE4 cells. The dilution factor causing the cytopathic effect was determined and the results were presented with standard deviation of independent experiments after normalization by that of wild type adenovirus. The results are normalized by wild-type adenovirus.

Abbreviation: Ad, adenovirus; mRFP, monomeric red fluorescence protein; ttk, modified herpes simplex virus thymidine kinase; PSES, prostate-specific enhancer sequence. 


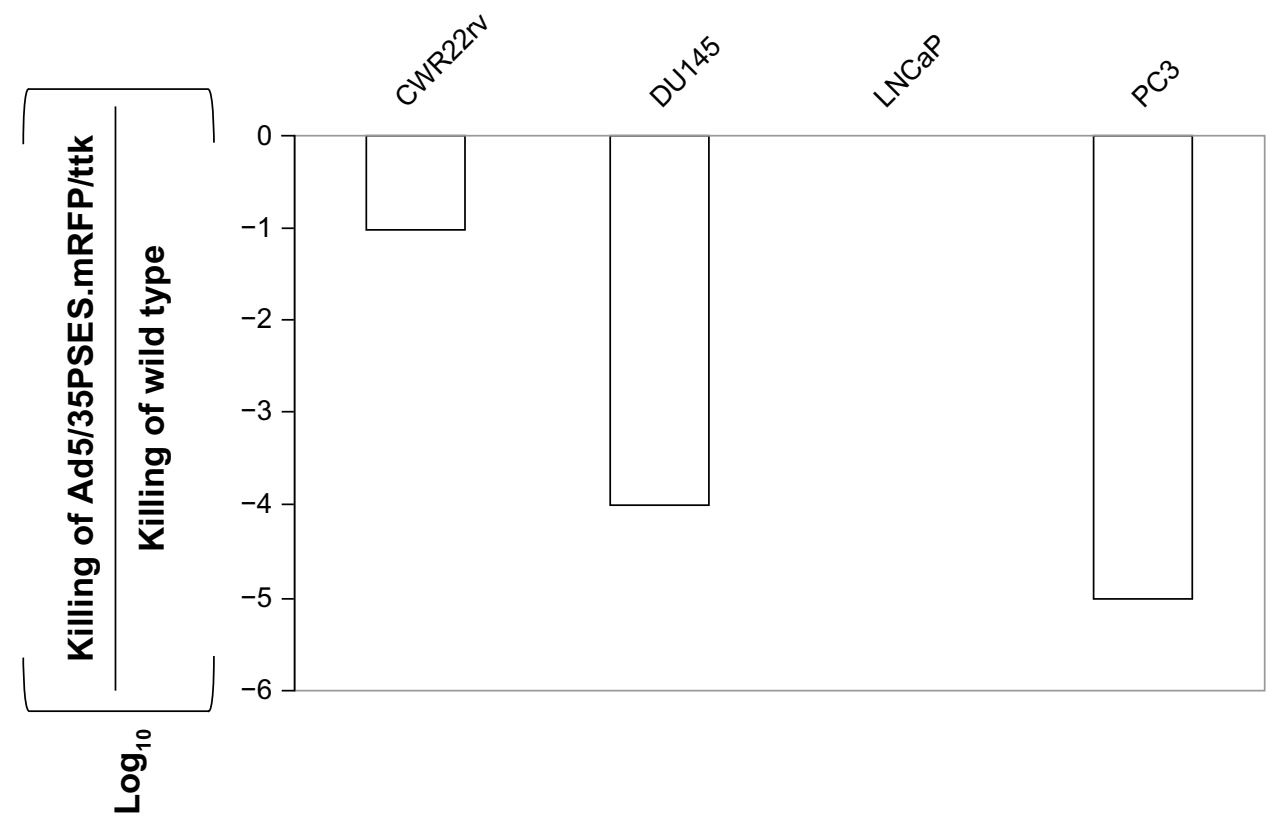

Figure 5 Tumor-specific killing activity of recombinant adenovirus. Viral killing activity is shown as the $\mathrm{LD}_{50}$. $\mathrm{A}$ tissue/tumor-specific killing index was obtained by dividing the $\mathrm{LD}_{50}$ of therapeutic viruses by that of wild-type adenovirus.

Abbreviations: Ad, adenovirus; mRFP, monomeric red fluorescence protein; ttk, modified herpes simplex virus thymidine kinase; $\mathrm{LD}_{50}$, $50 \%$ lethal dose; PSES, prostatespecific enhancer sequence.

cancerous and noncancerous aspects of the prostate is a viable approach; several prostate-specific promoters have been developed; and therapeutic genes can be expressed in a prostate-specific manner.

In general, two types of tissue/tumor-restricted replicative adenoviruses are under clinical investigation. The first is originally derived from E1b-deleted adenovirus known as ONYX-15. Adenoviral E1b protein can inactivate the tumor suppressor p53, allowing the virus to replicate in cells with functional $\mathrm{p} 53$. Without E1b proteins, ONYX-15 is expected to replicate only in p53-defective tumor cells but not in normal cells. Recently, another mechanism was reported to

\section{A}

\section{Ad5/35mFLT3L.PSES.mRFP/ttk}

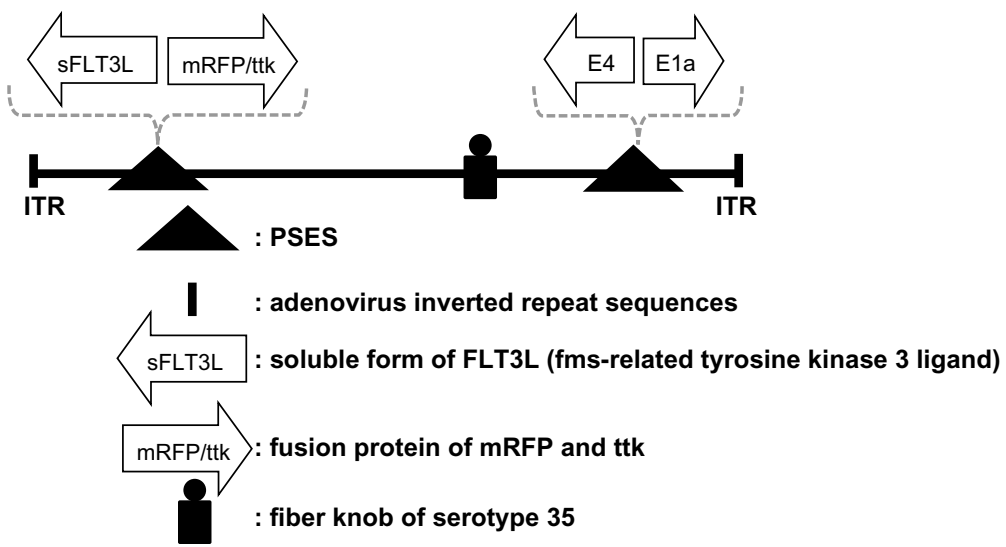

B

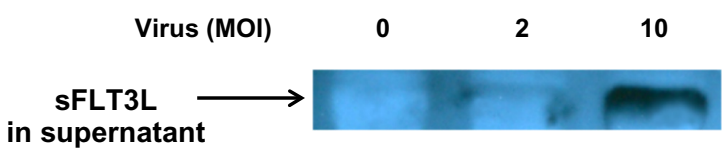

Figure 6 The new therapeutic adenovirus was constructed, as modified from recombinant adenovirus Ad5/35sFLT3L.PSES.mRFP/ttk. (A) A schematic diagram of adenovirus constructs is shown. Prostate-specific promoter (PSES) controls both $\mathrm{mFLT} 3 \mathrm{~L}$ and mRFP/ttk fusion proteins. (B) LNCaP cells at a density of $4 \times 10^{5}$ were infected with the indicated adenovirus as a multiplicity of infection (MOI) for 48 hours, followed by lysis in radioimmunoprecipitation assay buffer and Western blot analysis. sFLT3L expression by Ad5 mTR.sPDI (total of $80 \mu \mathrm{g}$ protein per lane) in the supernatant was confirmed using an anti-FLT3L antibody.

Abbreviations: Ad, adenovirus; ITR, intron; PSES, prostate specific enhancer sequence; mRFP, monomeric red fluorescence protein; ttk, modified herpes simplex virus thymidine kinase; FLT3L, fms-related tyrosine kinase 3 ligand. 
explain how ONYX-15 virus could differentiate tumor cells from normal cells. Some cancer cells support late E1b protein functions, such as preferential export and translation of late viral mRNAs. ${ }^{9,10}$ The second strategy is to control expression of adenovirus E1a genes by a tissue/tumor-specific enhancer/ promoter. Theoretically, without E1a proteins, expression of late gene proteins is diminished and the virus cannot propagate in the cell. Placing the E1a gene under the control of a tissue/tumor promoter should eventually control virus replication in a tissue/tumor-specific manner. This strategy was used to restrict adenovirus replication in hepatocellular and prostate carcinomas via alpha-fetoprotein and PSA promoters. ${ }^{11,12}$ There have been a number of studies utilizing tissue/tumor-restricted replicative adenovirus derived from either of two prototypes and developed further in clinical trials. Such approaches have thus become a very attractive potential strategy for prostate cancer.

All adenoviruses have a linear, double-stranded DNA genome. The genome is enclosed by capsids, which are composed of structural proteins including hexons and pentons, which bind to $\alpha_{\mathrm{v}} \beta_{3}$ and $\alpha_{\mathrm{v}} \beta_{5}$ integrins and proceed to virus internalization, and fiber. Attachment of adenovirus to host cells is initiated by a fiber knob, which projects outward from the penton base and binds to its high affinity receptor, CAR. ${ }^{13,14}$ Some studies have reported ineffective adsorption of adenovirus to cancer cells, due to the fact that CAR expression is downregulated in cancer, and urged researchers to modify the fiber knob to make adenovirus infection CARindependent. ${ }^{15-17}$ Unlike some cancer cells, recent studies have demonstrated that CAR is strongly expressed in metastatic prostate cancer, suggesting that adenovirus therapy might be a successful delivery tool for the treatment of prostate cancer. $^{18,19}$

In this study, we made a hybrid virus which had the serotype 35 fiber knob and entered cells independently of $\mathrm{CAR}^{20}$ to overcome the problem of CAR deficiency in tumor cells. Serotype 35 adenovirus binds to the CD46 receptor, which is also universally expressed on both normal and cancer cells. Once adenovirus binds to the receptors, it has the following life cycle: virus internalization after binding to CAR and integrin receptors; S-phase entry into the host cell; suppression of host cell apoptosis and protein synthesis; viral genome replication; viral structural protein synthesis; virion assembly in host cell nucleus; and host cell lysis and virion particle release. Oncolytic adenovirus therapy has attracted a lot of research attention as a novel anticancer strategy, and its natural cytotoxicity has been exploited to kill tumor cells directly. Recently, use of oncolytic adenovirus alone or in combination with other conventional treatment modalities, ie, chemotherapy or radiotherapy, has been shown to have potential as a therapeutic tool. ${ }^{21,22}$ However, results from preclinical studies suggest that new strategies should be developed to maximize oncolytic efficacy without risking safety. Therefore, in this study, we introduced a novel therapeutic adenovirus expressing $\mathrm{mRFP} / \mathrm{ttk}$ fusion proteins, activated both $\mathrm{mRFP} / \mathrm{ttk}$ and FLT3L cytokine. Expression of $\mathrm{mRFP} / \mathrm{ttk}$ fusion protein was tightly controlled by PSES, which is known to be active only in PSA-positive and PSMA-positive prostate cancer cells. Although we showed increasing red fluorescence intensity in a time-dependent manner in LNCaP cells, we produced the same results in CWR22rv cells. Not only was fluorescence detected in LNCaP and CWR22rv cells, but also thymidine kinase activity was measurable only in these cells. Unlike in $\mathrm{LNCaP}$ and $\mathrm{CWR} 22 \mathrm{rv}$ cells $\mathrm{mRFP} / \mathrm{ttk}$ fusion proteins were not expressed in DU145 and PC3 cells throughout this study. We chose FLT3L as an adjuvant cytokine because it is able to enhance the antitumor activity of thymidine kinase. ${ }^{2}$ To make antigen-presenting cells capable of antigen presentation to naive $\mathrm{T}$ cells, nonactivated dendritic cells must first be activated into mature dendritic cells. FLT3L plays a role in differentiation and expansion of these dendritic cells in vivo. ${ }^{23-27}$ However, once exposed to activated dendritic cells, naive T lymphocytes usually die prematurely in the absence of costimulatory molecules. Such a scenario enables the recombinant adenovirus constructed in this study to be a strong antitumoral agent; because mRFP/ttk can elicit cytotoxicity in tumor cells, the tumor cells that are destroyed would produce tumor antigens that can be used to activate dendritic cells in situ when infected with recombinant adenovirus. In this study, we used a chimeric recombinant adenovirus with a substantial advantage over serotype 5 , which is the adenovirus most commonly used for development of a gene therapy vector.

We developed two replication-competent adenoviruses controlled by the PSES promoter and showed that a single PSES promoter achieves prostate cancer-restricted control of adenoviral replication. In addition, the PSES promoter mediates the therapeutic mRFP/ttk gene in the right arm of the adenoviral genome. These adenoviruses were observed to replicate as efficiently as wild-type adenovirus in both hormone-dependent and hormone-independent prostate cancer cells. These promising results warrant further development of PSES promoter-based oncolytic adenovirus equipped with immunotherapeutic cytokines.

\section{Disclosure}

The authors report no conflicts of interest in this work. 


\section{References}

1. Annual report of cancer statistics in Korea in 2008. Ministry of Health and Welfare. 2010:19-31.

2. Ali S, King GD, Curtin JF, et al. Combined immunostimulation and conditional cytotoxic gene therapy provide long-term survival in a large glioma model. Cancer Res. 2005;65:7194-7204.

3. Lee SJ, Kim HS, Yu R, et al. Novel prostate-specific promoter derived from PSA and PSMA enhancers. Mol Ther. 2002;6:415-421.

4. Graham FL. Growth of 293 cells in suspension culture. J Gen Virol. 1987;68 Pt 3:937-940.

5. Li X, Zhang YP, Kim HS, et al. Gene therapy for prostate cancer by controlling adenovirus E1a and E4 gene expression with PSES enhancer. Cancer Res. 2005;65:1941-1951.

6. Kasono K, Blackwell JL, Douglas JT, et al. Selective gene delivery to head and neck cancer cells via an integrin targeted adenoviral vector. Clin Cancer Res. 1999;5:2571-2579.

7. Okegawa T, Li Y, Pong RC, Bergelson JM, Zhou J, Hsieh JT. The dual impact of coxsackie and adenovirus receptor expression on human prostate cancer gene therapy. Cancer Res. 2000;60:5031-5036.

8. Gardner TA, Sloan J, Raikwar SP, Kao C. Prostate cancer gene therapy: past experiences and future promise. Cancer Metastasis Rev. 2002;21:137-145.

9. O'Shea CC, Soria C, Bagus B, McCormick F. Heat shock phenocopies E1B-55K late functions and selectively sensitizes refractory tumor cells to ONYX-015 oncolytic viral therapy. Cancer Cell. 2005;8: 61-74.

10. O'Shea CC, Johnson L, Bagus B, et al. Late viral RNA export, rather than p53 inactivation, determines ONYX-015 tumor selectivity. Cancer Cell. 2004;6:611-623.

11. Hallenbeck PL, Chang YN, Hay C, et al. A novel tumor-specific replication-restricted adenoviral vector for gene therapy of hepatocellular carcinoma. Hum Gene Ther. 1999;10:1721-1733.

12. Rodriguez R, Schuur ER, Lim HY, Henderson GA, Simons JW, Henderson DR. Prostate attenuated replication competent adenovirus (ARCA) CN706: a selective cytotoxic for prostate-specific antigen-positive prostate cancer cells. Cancer Res. 1997;57: 2559-2563.

13. Bergelson JM, Cunningham JA, Droguett G, et al. Isolation of a common receptor for Coxsackie B viruses and adenoviruses 2 and 5. Science. 1997;275:1320-1323.

14. Tomko RP, Xu R, Philipson L. HCAR and MCAR: the human and mouse cellular receptors for subgroup $\mathrm{C}$ adenoviruses and group $\mathrm{B}$ coxsackieviruses. Proc Natl Acad Sci U S A. 1997;94:3352-3356.
15. Cripe TP, Dunphy EJ, Holub AD, et al. Fiber knob modifications overcome low, heterogeneous expression of the coxsackievirusadenovirus receptor that limits adenovirus gene transfer and oncolysis for human rhabdomyosarcoma cells. Cancer Res. 2001;61:2953-2960.

16. Li D, Duan L, Freimuth P, O'Malley BW Jr. Variability of adenovirus receptor density influences gene transfer efficiency and therapeutic response in head and neck cancer. Clin Cancer Res. 1999;5:4175-4181.

17. Li Y, Pong RC, Bergelson JM, et al. Loss of adenoviral receptor expression in human bladder cancer cells: a potential impact on the efficacy of gene therapy. Cancer Res. 1999;59:325-330.

18. Kubo H, Gardner TA, Wada Y, et al. Phase I dose escalation clinical trial of adenovirus vector carrying osteocalcin promoter-driven herpes simplex virus thymidine kinase in localized and metastatic hormonerefractory prostate cancer. Hum Gene Ther. 2003;14:227-241.

19. Rauen KA, Sudilovsky D, Le JL, et al. Expression of the coxsackie adenovirus receptor in normal prostate and in primary and metastatic prostate carcinoma: potential relevance to gene therapy. Cancer Res. 2002;62:3812-3818.

20. Gaggar A, Shayakhmetov DM, Lieber A. CD46 is a cellular receptor for group B adenoviruses. Nat Med. 2003;9:1408-1412.

21. Johanson TJ, Höti N, Liu C, et al. Bicalutamide-activated oncolytic adenovirus for the adjuvant therapy of high-risk prostate cancer. Cancer Gene Ther. 2013;20:394-402.

22. Zhang Z, Zhang X, Newman K, Liu X. MicroRNA regulation of oncolytic adenovirus 6 for selective treatment of castration-resistant prostate cancer. Mol Cancer Ther. 2012;11:2410-2418.

23. Maraskovsky E, Brasel K, Teepe M, et al. Dramatic increase in the numbers of functionally mature dendritic cells in Flt3 ligand-treated mice: multiple dendritic cell subpopulations identified. J Exp Med. 1996;184:1953-1962.

24. Parajuli P, Mosley RL, Pisarev V, et al. Flt3 ligand and granulocytemacrophage colony-stimulating factor preferentially expand and stimulate different dendritic and T-cell subsets. Exp Hematol. 2001;29: 1185-1193.

25. Masten BJ, Olson GK, Kusewitt DF, Lipscomb MF. Flt3 ligand preferentially increases the number of functionally active myeloid dendritic cells in the lungs of mice. J Immunol. 2004;172:4077-4408.

26. McKenna HJ, Stocking KL, Miller RE, et al. Mice lacking flt3 ligand have deficient hematopoiesis affecting hematopoietic progenitor cells, dendritic cells, and natural killer cells. Blood. 2000;95:3489-3497.

27. Shurin GV, Chatta GS, Tourkova IL, Zorina TD, Esche C, Shurin MR. Regulation of dendritic cell expansion in aged athymic nude mice by FLT3 ligand. Exp Gerontol. 2004;39:339-348.
OncoTargets and Therapy

\section{Publish your work in this journal}

OncoTargets and Therapy is an international, peer-reviewed, open access journal focusing on the pathological basis of all cancers, potential targets for therapy and treatment protocols employed to improve the management of cancer patients. The journal also focuses on the impact of management programs and new therapeutic agents and protocols on
Dovepress

patient perspectives such as quality of life, adherence and satisfaction The manuscript management system is completely online and includes a very quick and fair peer-review system, which is all easy to use. Visit $\mathrm{http}: / /$ www.dovepress.com/testimonials.php to read real quotes from published authors. 